\title{
Waring's problem with small prime factors
}

\author{
by
}

\author{
Gergely Harcos (Budapest)
}

1. Introduction. As a natural continuation of the results of Hardy, Littlewood and Vinogradov, much attention has been paid in the last 60 years to the Waring-Goldbach problem, the problem of representing natural numbers as a sum of prime powers. This is the main subject of Hua's book [7]. Stronger results can be drawn if one restricts to almost prime powers only. For example, by results of Brüdern and Fouvry, every sufficiently large integer in some congruence class can be represented as a sum of four squares all having at most 34 prime factors [3] and as a sum of seven cubes all having at most 69 prime factors [2].

However, it is plausible to expect similar results if other multiplicative constraints are imposed on the variables. In the light of the WaringGoldbach problem it is reasonable to study Waring's problem in variables which have relatively small prime factors (such numbers are often called smooth by a terminology of Pomerance). This problem has been studied only in the case of first powers so far (Balog and Sárközy [1]), this special case arising from a conjecture of Erdős.

The present paper tries to fill this gap by solving the general Waring problem in smooth numbers. While the main goal of this paper was to guarantee solutions with prime factors as small as possible and the number of summands $s$ was only of a secondary importance in this work, it turned out that even with the best result for the prime factors yielded by the method, one can bound $s$ in the same way, at least when $k$ is large, as available in the classical Waring problem at present [10]. More precisely, using the notations of Wooley [10, Theorem 1.2] and writing $P(n)$ for the greatest prime factor of a number $n$ we shall prove

TheOREM. For any positive integer $k$ there exist a positive integer $s$ and positive constants $c_{1}, c_{2}$ such that every sufficiently large integer $N$ has at least

$$
N^{s / k-1} \exp \left(-c_{1}(\log N \log \log N)^{1 / 2}\right)
$$


representations of the form

$$
n_{1}^{k}+n_{2}^{k}+\ldots+n_{s}^{k}=N
$$

where

$$
P\left(n_{1} n_{2} \ldots n_{s}\right) \leq \exp \left(c_{2}(\log N \log \log N)^{1 / 2}\right) .
$$

Moreover, the least such $s$ satisfies $s \leq F(k)$ where $F(1)=3, F(2)=5$, $F(3)=9, F(4)=17, F(5)=21, F(6)=29, F(7)=39, F(8)=49$ and, for $k \geq 9$,

$$
F(k)=3+2 \min _{v \geq k+1}\left(v+\left[\frac{\Delta(v)}{2 \varrho}\right]\right) .
$$

In particular, as $k$ tends to infinity,

$$
F(k) \leq k(\log k+\log \log k+O(1)) .
$$

Remark 1 . The results are probably not the best ones. However, qualitatively they are as sharp as is known in the special case of the first powers [1]. To indicate the strength of the Theorem we note that among the first $N$ positive integers the proportion of the numbers composed of prime factors not exceeding $\exp \left((\log N)^{1 / 2+o(1)}\right)$ is $\exp \left(-(\log N)^{1 / 2+o(1)}\right)$, as follows from the work of de Bruijn [4] and Hildebrand [5] (cf. [6, Corollary 1.3]). The bound for the size of the prime factors cannot be replaced with any function of order of magnitude $(\log N)^{1+o(1)}$ in our Theorem. In fact, in this case [4] would yield that we had only a set of cardinality $N^{o(1)}$ to choose the $k$ th powers from in order to represent all sufficiently large positive integers $n$ up to $N$, contradicting the consequence of a simple counting argument, namely that, given $s$, at least $N^{1 / s-o(1)} k$ th powers are needed.

R e mark 2. Observe that for $k \geq 21, F(k)$ is the best known bound for $s$ in the classical Waring problem due to Wooley [10]. The first 20 values of $F(k)$ are listed in the following table.

\begin{tabular}{ccccccccccc}
\hline$k$ & 1 & 2 & 3 & 4 & 5 & 6 & 7 & 8 & 9 & 10 \\
$F(k)$ & 3 & 5 & 9 & 17 & 21 & 29 & 39 & 49 & 57 & 65 \\
\hline \multicolumn{1}{c}{} & & & & & & & & & & \\
\hline$k$ & 11 & 12 & 13 & 14 & 15 & 16 & 17 & 18 & 19 & 20 \\
$F(k)$ & 73 & 81 & 89 & 97 & 105 & 115 & 123 & 131 & 139 & 149 \\
\hline
\end{tabular}

R e m a r k 3 . The proof yields effectively computable good values of $c_{1}, c_{2}$ in terms of $s$ and $k$. For example, $(1,3,2 / 9,15)$ or $(2,5,2 / 9,24)$ are admissible as $\left(k, s, c_{1}, c_{2}\right)$. Also, a similar result could be shown for any $s \geq F(k)$.

Acknowledgements. I would like to thank Professor A. Sárközy for calling my attention to this problem, his encouragement and valuable sug- 
gestions during the work. I am grateful to Professor A. Balog, who believed at an early stage of the results that they might well be improved significantly. Let me express my gratitude also to the anonymous referee, who pointed out that smooth numbers might provide a better version of my original Lemma 11. This comment led to the present good bound on $s$.

2. Notations and preliminary remarks. In order to prove our Theorem, we use the Hardy-Littlewood method combining with other ideas similar to those of [1], some strong results of [9] and [10] on the classical Waring problem and a new inequality concerning the number of a certain type of divisors of a number. If $k \geq 9$ then we define the $\Delta(v)$ 's and $\varrho$ in terms of $k$ as in [10, Theorem 1.2]. We record for later reference that $\varrho<1 /(2 k)$ follows at once from these definitions. $F(k)$ is the function in our Theorem.

We write $e^{x}=\exp (x)$ and $e^{2 \pi i \alpha}=e(\alpha)$. The distance from $x$ to the nearest integer is denoted by $\|x\|$. We define the empty sum to be 0 and the empty product to be 1 . We put $\min \left(A, \frac{1}{0}\right)=A$. We denote the least prime factor of $n$ by $p(n)$, while the greatest prime factor of $n$ is denoted by $P(n) . \tau(n)$ denotes the divisor function $\sum_{m \mid n} 1$, and $\Omega(n)=\sum_{p^{\alpha} \| n} \alpha$ counts the number of prime factors of $n$ with multiplicity. The Vinogradov symbols $\ll, \gg$ have their usual meaning, namely that for functions $f$ and $g$ with $g$ taking non-negative values $f \ll g$ and $g \gg f$ means $|f| \leq C g$ where $C$ is a constant. The dependence of the implicit constants in the $O, \ll$ and $\gg$ notations, if any, will be indicated explicitly in the subscripts of these symbols.

We shall always assume, without any remark, that $N$ is sufficiently large to fit in our statements. Whenever we use the $o$-symbol without any comment, we understand it as $N \rightarrow \infty$. Let $s$ be a positive integer depending only on $k$ which we specify later. Define $w=\exp \left(\left(\log N^{1 / k} \log \log N^{1 / k}\right)^{1 / 2}\right)$, and put $y=w^{c_{3}}$ and $z=y^{2 / 9}$ where $c_{3}$ is a positive constant to be chosen later in terms of $k$ and $s$. Let

$$
\begin{gathered}
Q=\frac{N}{z^{1 / 4}}, \quad U=\left[2 k \frac{N}{y}\right]+1, \\
\mathcal{L}=\left\{l: \lambda \frac{N^{1 / k}}{y} \leq l \leq \frac{N^{1 / k}}{y} \text { and } z<p(l) \leq P(l) \leq y\right\}
\end{gathered}
$$

with an appropriate $0<\lambda<1$ to be determined later in terms of $k$. Note that $\mathcal{L}$ is not empty, since $y=o\left(N^{1 / k}\right)$. We write

$$
L=\sum_{l \in \mathcal{L}} 1, \quad d_{n}=\sum_{\substack{m l=n \\ m \leq y \\ l \in \mathcal{L}}} 1 \quad\left(\text { for } 1 \leq n \leq N^{1 / k}\right)
$$




$$
f(\alpha)=\sum_{1 \leq n \leq N^{1 / k}} d_{n} e\left(n^{k} \alpha\right), \quad u(\alpha)=\frac{1}{U} \sum_{n=0}^{U-1} e(n \alpha)
$$

and

$$
h(\alpha)=f(\alpha) u(\alpha)=\sum_{n=1}^{N+U-1} h_{n} e(n \alpha)
$$

so that

$$
h_{n}=\frac{1}{U} \sum_{n-U<j^{k} \leq n} d_{j} .
$$

We shall study the integral

$$
J=\int_{0}^{1} f(\alpha)^{s} e(-N \alpha) d \alpha=\sum_{n_{1}^{k}+\ldots+n_{s}^{k}=N} d_{n_{1}} \ldots d_{n_{s}}
$$

by comparing it to

$$
I=\int_{0}^{1} h(\alpha)^{s} e(-N \alpha) d \alpha=\sum_{n_{1}+\ldots+n_{s}=N} h_{n_{1}} \ldots h_{n_{s}} .
$$

The integrals provide weighted enumerations of representations of $N$ as a sum of $s$ positive integers. The main feature of the weights $d_{n}$ is that $d_{n}>0$ implies $P(n) \leq y$, while the advantage of the averaged weights $h_{n}$ lies in the expectation that their discrepancies are smaller than those of the $d_{n}$ 's. In fact, it is quite straightforward to give a lower estimation for $h_{n}$ and the integral $I$. However, to treat $J$ and $I$ properly we need an upper estimate for the quantities $d_{n}$. We note that the weights $d_{n}$ were invented by Balog and Sárközy [1], who later derived several theorems using them. Almost all of these results become stronger when the new inequality, our Lemma 3, is taken into account.

To prove our Theorem, we shall need several lemmata which we collect in the next section. It will be convenient to work on the unit interval $\mathfrak{U}=$ $[1 / Q, 1+1 / Q]$ instead of $[0,1]$. Note that the generating functions $e(\alpha), f(\alpha)$, $u(\alpha)$ and $h(\alpha)$ appearing here are all periodic modulo 1 , hence they depend only on the fractional part of $\alpha$. For any $1 \leq a \leq q \leq z$ and $(a, q)=1$ introduce the intervals

$$
\mathfrak{M}(q, a)=\left\{\alpha:\left|\alpha-\frac{a}{q}\right| \leq \frac{1}{Q}\right\},
$$

which are pairwise disjoint and lie in $\mathfrak{U}$. Hence if we define the major arcs $\mathfrak{M}$ as the union of these intervals and the minor arcs as $\mathfrak{m}=\mathfrak{U} \backslash \mathfrak{M}$, then clearly $\mathfrak{U}$ is the disjoint union of $\mathfrak{M}$ and $\mathfrak{m}$. 
In accordance with [9] and [10], for any $2 \leq R \leq P$ define

$$
\mathcal{A}(P, R)=\{n: n \leq P \text { and } P(n) \leq R\} .
$$

Then, evidently, $\mathcal{L} \subseteq \mathcal{A}\left(N^{1 / k} / y, y\right)$. Let $S_{t}(P, R)$ denote the number of solutions of

$$
x_{1}^{k}+\ldots+x_{t}^{k}=y_{1}^{k}+\ldots+y_{t}^{k}
$$

with

$$
x_{1}, \ldots, x_{t}, y_{1}, \ldots, y_{t} \in \mathcal{A}(P, R) .
$$

Finally, we define the broadly used exponential sums

$$
S(q, a)=\sum_{r=1}^{q} e\left(r^{k} \frac{a}{q}\right) .
$$

3. Preliminary lemmata. First of all let us formulate a simple lemma for further reference and also recall some classical estimates for the divisor function $\tau$.

LEMMA 1. If $a, b$ are real numbers and $m$ is a positive integer then

$$
\left|a^{m}-b^{m}\right| \leq m|a-b| \max \left(|a|^{m-1},|b|^{m-1}\right) .
$$

Proof. The equality

$$
\left|a^{m}-b^{m}\right|=|a-b|\left|\sum_{j=0}^{m-1} a^{j} b^{m-1-j}\right|
$$

yields the result.

Lemma 2. We have, for every positive integer $m$,

$$
\sum_{1 \leq u \leq P} \tau(u)^{m} \leq P(\log 3 P)^{2^{m}-1},
$$

and, as $P \rightarrow \infty$,

$$
\max _{1 \leq u \leq P} \tau(u) \leq P^{o(1)} .
$$

P r o of. The first part is an explicit version of [7, Hilfssatz 2.5] admitting the same proof. The second part is an immediate consequence of the first part.

The following lemma is an essential tool in the paper.

LEMMA 3. We have

$$
\max _{1 \leq n \leq N^{1 / k}} d_{n} \leq w^{o(1)}
$$


Proof. For all $n$ define $n^{-}$and $n^{+}$as

$$
n^{-}=\prod_{\substack{p^{\alpha} \| n \\ p \leq z}} p^{\alpha} \quad \text { and } \quad n^{+}=\prod_{\substack{p^{\alpha} \| n \\ p>z}} p^{\alpha} .
$$

It is clear that $n=n^{-} n^{+}$and $m \mid n$ if and only if $m^{-} \mid n^{-}$and $m^{+} \mid n^{+}$.

Take any $1 \leq n \leq N^{1 / k}$ with $d_{n}>0$. Then $n$ can be written as $m_{0} l_{0}$ where $m_{0} \leq y$ and $l_{0} \in \mathcal{L}$. Fix $n, m_{0}, l_{0}$ for the rest of the proof. The definitions of $n^{+}$and $\mathcal{L}$ show that $l_{0} \mid n^{+}$, therefore $n^{-} \mid m_{0}$ at the same time. In particular, $n^{-} \leq y$. Any representation $n=m l$ contributing to $d_{n}$ is determined by the divisor $m \mid n$ satisfying $m \leq y$. This $m$ in turn is determined by the pair of divisors $m^{-} \mid n^{-}$and $m^{+} \mid n^{+}$. The number of choices for $m^{-}$is

$$
\tau\left(n^{-}\right) \leq \max _{1 \leq u \leq y} \tau(u)
$$

Now consider $m^{+}$. By definition, we know that

$$
z<p\left(n^{+}\right) \leq p\left(m^{+}\right) \leq m^{+} \leq m \leq y<z^{5}
$$

from which it follows that $m^{+}$has at most 4 prime factors. These prime factors are among those of $n^{+}$, therefore the number of choices for $m^{+}$is at most

$$
\sum_{r=0}^{4}\left(\begin{array}{c}
\Omega\left(n^{+}\right) \\
r
\end{array}\right) \ll \Omega\left(n^{+}\right)^{4} \leq\left(\frac{\log n^{+}}{\log 2}\right)^{4} \ll\left(\log n^{+}\right)^{4} \leq(\log N)^{4} .
$$

Combining our results with the second part of Lemma 2 we can see that

$$
\max _{1 \leq n \leq N^{1 / k}} d_{n} \ll(\log N)^{4} \max _{1 \leq u \leq y} \tau(u) \leq w^{o(1)} y^{o(1)}=w^{o(1)} .
$$

As a counterpart to the previous lemma, we state

LEMMA 4. We have

$$
\min _{U \leq n \leq \lambda^{k} N} h_{n} \geq L / U
$$

Proof. Let $U \leq n \leq \lambda^{k} N$. Then

$$
\begin{aligned}
U h_{n} & =\sum_{n-U<j^{k} \leq n} d_{j}=\sum_{n-U<j^{k} \leq n} \sum_{\substack{m l=j \\
m \leq y \\
l \in \mathcal{L}}} 1 \\
& =\sum_{\substack{n-U<m^{k} l^{k} \leq n \\
m \leq \mathcal{y} \\
l \in \mathcal{L}}} 1=\sum_{\substack{l \in \mathcal{L} \\
(n-U) / l^{k}<m^{k} \leq n / l^{k} \\
m \leq y}} 1 .
\end{aligned}
$$


As $\lambda N^{1 / k} / y \leq l$ holds for every $l \in \mathcal{L}$, also

$$
\frac{n}{l^{k}} \leq \frac{\lambda^{k} N}{\lambda^{k} N / y^{k}}=y^{k} \text {. }
$$

This means that we can drop the condition $m \leq y$ in the inner sum, i.e., using also Lemma 1,

$$
\begin{aligned}
U h_{n} & =\sum_{l \in \mathcal{L}} \sum_{(n-U) / l^{k}<m^{k} \leq n / l^{k}} 1 \\
& \geq \sum_{l \in \mathcal{L}}\left(\frac{\sqrt[k]{n}-\sqrt[k]{n-U}}{l}-1\right) \geq \sum_{l \in \mathcal{L}}\left(\frac{U}{k n^{1-1 / k} l}-1\right) \\
& \geq \sum_{l \in \mathcal{L}}\left(\frac{U}{k n^{1-1 / k} l}-\frac{U}{2 k N / y}\right) \geq \sum_{l \in \mathcal{L}}\left(\frac{U}{k n^{1-1 / k} l}-\frac{U}{2 k n^{1-1 / k} l}\right) \\
& =\sum_{l \in \mathcal{L}} \frac{U}{2 k n^{1-1 / k} l} \geq \sum_{l \in \mathcal{L}} \frac{2 k N / y}{2 k n^{1-1 / k} N^{1 / k} / y}=L\left(\frac{N}{n}\right)^{1-1 / k} \geq L .
\end{aligned}
$$

In order to use this lemma, we need a lower estimate for $L$.

LEMma 5. We have

$$
L \geq \frac{N^{1 / k}}{y} w^{-1 / c_{3}-o(1)} .
$$

Proof. Since $z \leq \frac{1}{2} y^{1 / 2}$, we can follow the proof of [1, Lemmata 13 and 14] step by step to deduce that

$$
L \geq \frac{N^{1 / k}}{y} \exp \left(-(1+o(1)) \frac{\log N^{1 / k} \log \log N^{1 / k}}{\log y}\right)
$$

which is the same as the statement of the lemma, since

$$
\frac{\log N^{1 / k} \log \log N^{1 / k}}{\log y}=\frac{(\log w)^{2}}{\log y}=\frac{(\log w)^{2}}{c_{3} \log w}=\frac{1}{c_{3}} \log w .
$$

For studying the generating functions $f$ and $h$ on the major arcs we shall need

Lemma 6. Let $a, q, M$ be positive integers, $\beta$ an arbitrary real number and $v$ a polynomial with positive integer coefficients. If

$$
g_{m}(\alpha)=\sum_{n=1}^{m} e(v(n) \alpha) \quad \text { and } \quad g(\alpha)=g_{M}(\alpha)
$$

then

$$
g\left(\frac{a}{q}+\beta\right)=\left(\frac{1}{q} \sum_{r=1}^{q} e\left(v(r) \frac{a}{q}\right)\right) g(\beta)+O(q(1+|\beta| v(M))) .
$$


Proof. For simplicity put $S=\sum_{r=1}^{q} e\left(v(r) \frac{a}{q}\right)$. For any integer $1 \leq m \leq$ $M$ we have

$$
g_{m}\left(\frac{a}{q}\right)=\sum_{n=1}^{m} e\left(v(n) \frac{a}{q}\right)=\sum_{j=0}^{[m / q]-1} \sum_{r=1}^{q} e\left(v(j q+r) \frac{a}{q}\right)+O(q)
$$

where $v(j q+r) \equiv v(r)(\bmod q)$, i.e.,

$$
g_{m}\left(\frac{a}{q}\right)=\left[\frac{m}{q}\right] \sum_{r=1}^{q} e\left(v(r) \frac{a}{q}\right)+O(q)=\frac{m}{q} S+O(q) .
$$

Using this result we get

$$
\begin{aligned}
g\left(\frac{a}{q}+\beta\right)= & \sum_{m=1}^{M} e\left(v(m) \frac{a}{q}\right) e(v(m) \beta) \\
= & \sum_{m=1}^{M}\left(g_{m}\left(\frac{a}{q}\right)-g_{m-1}\left(\frac{a}{q}\right)\right) e(v(m) \beta) \\
= & g_{M}\left(\frac{a}{q}\right) e(v(M) \beta)+\sum_{m=1}^{M-1} g_{m}\left(\frac{a}{q}\right)(e(v(m) \beta)-e(v(m+1) \beta)) \\
= & \left(\frac{M}{q} S+O(q)\right) e(v(M) \beta) \\
& +\sum_{m=1}^{M-1}\left(\frac{m}{q} S+O(q)\right)(e(v(m) \beta)-e(v(m+1) \beta)) \\
= & \sum_{m=1}^{M}\left(\frac{m}{q} S-\frac{m-1}{q} S\right) e(v(m) \beta) \\
& +O(q)\left\{1+|\beta| \sum_{m=1}^{M-1}|v(m)-v(m+1)|\right\} \\
= & \frac{1}{q} S g(\beta)+O(q(1+|\beta| v(M))),
\end{aligned}
$$

since $v$ is an increasing function on the positive integers.

The treatment of the minor arcs will require the use of the following well-known result.

Lemma 7. If $\alpha, M, V$ are real numbers and $a, q$ are integers such that $M \geq 1, q>0,(a, q)=1$ and $|\alpha-a / q| \leq 1 / q^{2}$ then

$$
\sum_{1 \leq m \leq M} \min \left(V, \frac{1}{2\|m \alpha\|}\right) \leq\left(\frac{M}{q}+1\right)(6 V+q \log q) .
$$


Proof. This follows at once from [7, Hilfssatz 3.5], as any interval of length $M$ can be covered by $[M / q]+1$ intervals of length $q$.

\section{Estimating the generating functions}

LEMma 8. We have, for all $\alpha$,

$$
|f(\alpha)| \leq \text { Ly } \quad \text { and } \quad|u(\alpha)| \leq 1 .
$$

Proof. This is obvious by estimating the terms in $f(\alpha)$ and $u(\alpha)$ trivially.

Lemma 9. We have, for all $\alpha$,

$$
|u(\alpha)-1| \leq \pi|\alpha| U .
$$

Proof. This follows from [1, Lemma 1].

The behaviour of the generating functions $f$ and $h$ on the major arcs is described by

Lemma 10. If $\alpha \in \mathfrak{M}$, e.g. $\alpha \in \mathfrak{M}(q, a)$ then

$$
f(\alpha)=q^{-1} S(q, a) f(\beta)+O\left(L z^{2}\right), \quad h(\alpha)=\delta_{q} h(\beta)+O\left(L z^{2}\right)
$$

where $\beta=\alpha-a / q$ and $\delta_{q}=1$ or 0 according as $q=1$ or $q>1$.

Proof. By the definition of $\mathfrak{M}$ we have $1 \leq a \leq q \leq z,(a, q)=1$ and $|\beta| \leq 1 / Q$. Now the proof is based on the fact that $q$ is relatively prime to all elements $l$ of $\mathcal{L}$, since $q \leq z<p(l)$. Therefore, applying Lemma 6 ,

$$
\begin{aligned}
f(\alpha) & =\sum_{\substack{m \leq y \\
l \in \mathcal{L}}} e\left(m^{k} l^{k} \alpha\right)=\sum_{l \in \mathcal{L}} \sum_{m \leq y} e\left(m^{k} l^{k} \alpha\right) \\
& =\sum_{l \in \mathcal{L}}\left\{q^{-1} S\left(q, l^{k} a\right) \sum_{m \leq y} e\left(m^{k} l^{k} \beta\right)+O\left(q\left(1+\left|l^{k} \beta\right| y^{k}\right)\right)\right\} .
\end{aligned}
$$

In the inner expression we have, for all $l \in \mathcal{L}$,

$$
S\left(q, l^{k} a\right)=\sum_{r=1}^{q} e\left((r l)^{k} \frac{a}{q}\right)=S(q, a)
$$

while in the error term

$$
q\left(1+\left|l^{k} \beta\right| y^{k}\right) \leq z\left(1+\frac{N}{y^{k}} \cdot \frac{1}{Q} y^{k}\right)<z(1+z) \leq 2 z^{2} .
$$

This implies that

$$
\begin{aligned}
f(\alpha) & =q^{-1} S(q, a) \sum_{l \in \mathcal{L}} \sum_{m \leq y} e\left(m^{k} l^{k} \beta\right)+O\left(L z^{2}\right) \\
& =q^{-1} S(q, a) f(\beta)+O\left(L z^{2}\right),
\end{aligned}
$$


the first part of the lemma. As to the other part, we use Lemma 6 once more to deduce

$$
u(\alpha)=\delta_{q} u(\beta)+O\left(\frac{1}{U} q(1+|\beta| U)\right)
$$

where $\delta_{q}=1$ or 0 according as $q=1$ or $q>1$ and

$$
\frac{1}{U} q(1+|\beta| U) \leq \frac{1}{U} z\left(1+\frac{U}{Q}\right)<\frac{2 z}{U},
$$

i.e.,

$$
u(\alpha)=\delta_{q} u(\beta)+O\left(\frac{z}{U}\right) .
$$

Putting this result together with the one we obtained for $f(\alpha)$ gives

$$
\begin{aligned}
h(\alpha) & =f(\alpha) u(\alpha) \\
& =\delta_{q} q^{-1} S(q, a) f(\beta) u(\beta)+O\left(|f(\beta)| \frac{z}{U}\right)+O\left(|u(\beta)| L z^{2}\right)+O\left(L z^{2} \frac{z}{U}\right)
\end{aligned}
$$

where we used the trivial estimate $\left|q^{-1} S(q, a)\right| \leq 1$. Now observe that

$$
\delta_{q} q^{-1} S(q, a)=\delta_{q}
$$

and the error terms sum up to $O\left(L z^{2}\right)$ by Lemma 8 in this expression. Hence

$$
h(\alpha)=\delta_{q} h(\beta)+O\left(L z^{2}\right)
$$

which is the second part of the lemma.

In order to estimate the generating functions on the minor arcs $\mathfrak{m}$ and to derive the upper bound $s \leq F(k)$ we need the following mean value inequality.

LEMMA 11. For any positive constant $c_{4}$ there exist a positive integer $t$ depending only on $k$ and a positive constant $c_{5}$ depending only on $k$ and $c_{4}$ such that

$$
S_{t}(P, R) \leq P^{2 t-k}(\log 2 P)^{c_{5}}
$$

holds for all $P \geq 2$ with $R=\exp \left(c_{4}(\log P \log \log P)^{1 / 2}\right)$. Moreover, if $t$ satisfies this property then so does $t+1$, and the least such $t$ satisfies $2 t \leq$ $F(k)-1$.

Pr o of. Introduce the generating functions

$$
l(\alpha)=\sum_{n \in \mathcal{A}(P, R)} e\left(n^{k} \alpha\right), \quad k(\alpha)=\sum_{1 \leq n \leq P} e\left(n^{k} \alpha\right) .
$$

The inequality in the lemma can be written as

$$
\int_{0}^{1}\left|l(\alpha)^{2 t}\right| d \alpha \leq P^{2 t-k}(\log 2 P)^{c_{5}},
$$


from which it is clear that the inequality still holds with $t+1$ in place of $t$, since

$$
\int_{0}^{1}\left|l(\alpha)^{2 t+2}\right| d \alpha \leq \max _{[0,1]}|l(\alpha)|^{2} \int_{0}^{1}\left|l(\alpha)^{2 t}\right| d \alpha \leq P^{2} \int_{0}^{1}\left|l(\alpha)^{2 t}\right| d \alpha .
$$

If $1 \leq k \leq 4$ then the existence of $2 t$ and the inequality for it can be simply deduced from Hua's inequality [7, Satz 4]. If $k \geq 5$ then put

$$
L=\int_{0}^{1}\left|l(\alpha)^{2 t}\right| d \alpha, \quad K=\int_{0}^{1}\left|k(\alpha)^{2} l(\alpha)^{2 t-2}\right| d \alpha .
$$

It is clear by considering the underlying diophantine equations that

$$
L \leq K \text {. }
$$

If $5 \leq k \leq 8$ and $2 t=F(k)-1$ then [9, Lemma 5.2] improved by the new estimates of $[10, \S 5]$ shows that

$$
K \ll_{k} P^{2 t-k}
$$

(cf. also $[10, \S 6]$ ), hence by (1) any sufficiently large $c_{5}>0$ (in terms of $k$ and $\left.c_{4}\right)$ is admissible in the lemma.

Suppose now that $k \geq 9$. For any $1 \leq a \leq q \leq P$ and $(a, q)=1$ introduce the intervals

$$
\mathfrak{N}(q, a)=\left\{\alpha:\left|\alpha-\frac{a}{q}\right| \leq \frac{P^{1-k}}{2 k q}\right\},
$$

which are pairwise disjoint and lie in the unit interval

$$
\mathfrak{V}=\left[\frac{P^{1-k}}{2 k}, 1+\frac{P^{1-k}}{2 k}\right] .
$$

Hence if we define $\mathfrak{N}$ as the union of these intervals and we put $\mathfrak{n}=\mathfrak{V} \backslash \mathfrak{N}$, then clearly $\mathfrak{V}$ is the disjoint union of $\mathfrak{N}$ and $\mathfrak{n}$, i.e.

$$
K=\int_{\mathfrak{N}}\left|k(\alpha)^{2} l(\alpha)^{2 t-2}\right| d \alpha+\int_{\mathfrak{n}}\left|k(\alpha)^{2} l(\alpha)^{2 t-2}\right| d \alpha .
$$

First, by Hölder's inequality,

$$
\int_{\mathfrak{N}}\left|k(\alpha)^{2} l(\alpha)^{2 t-2}\right| d \alpha \leq\left(\int_{\mathfrak{N}}\left|k(\alpha)^{2 t}\right| d \alpha\right)^{1 / t}\left(\int_{\mathfrak{N}}\left|l(\alpha)^{2 t}\right| d \alpha\right)^{1-1 / t} .
$$

If $2 t \geq k+2$ then [9, Lemma 5.1] implies

$$
\int_{\mathfrak{N}}\left|k(\alpha)^{2 t}\right| d \alpha \ll_{k, t} P^{2 t-k},
$$

and (1) yields

$$
\int_{\mathfrak{N}}\left|l(\alpha)^{2 t}\right| d \alpha \leq L \leq K
$$


therefore

$$
\int_{\mathfrak{N}}\left|k(\alpha)^{2} l(\alpha)^{2 t-2}\right| d \alpha \ll_{k, t}\left(P^{2 t-k}\right)^{1 / t} K^{1-1 / t} .
$$

Think now of $t$ as a sum $u+v$ of positive integers where $v \geq k+1$. Then condition $2 t \geq k+2$ is automatically satisfied, and we also see that

$$
\int_{\mathfrak{n}}\left|k(\alpha)^{2} l(\alpha)^{2 t-2}\right| d \alpha \leq\left(\sup _{\mathfrak{n}}|l(\alpha)|\right)^{2 u} \int_{0}^{1}\left|k(\alpha)^{2} l(\alpha)^{2 v-2}\right| d \alpha .
$$

Choose any constant $\varepsilon>0$. Since $\varrho<1 /(2 k)$, and $R=P^{o(1)}$ as $P \rightarrow \infty$, we can deduce from $[10$, Theorem 1.4] that

$$
\sup _{\mathfrak{n}}|l(\alpha)| \leq P^{1-\varrho+\varepsilon}
$$

while we learn from Wooley's remark in the last paragraph of $[10$, p. 150] that

$$
\int_{0}^{1}\left|k(\alpha)^{2} l(\alpha)^{2 v-2}\right| d \alpha \ll_{k, \varepsilon} P^{2 v-k+\Delta(v)+\varepsilon} .
$$

As $\varepsilon>0$ may be chosen arbitrarily small, we find that

$$
2 u \varrho>\Delta(v)
$$

implies

$$
\int_{\mathfrak{n}}\left|k(\alpha)^{2} l(\alpha)^{2 t-2}\right| d \alpha \ll_{k, t} P^{2 t-k} .
$$

This together with (2) and (3) gives

$$
K \ll_{k, t}\left(P^{2 t-k}\right)^{1 / t} K^{1-1 / t}+P^{2 t-k},
$$

so that, using also (1),

$$
L \leq K \ll_{k, t} P^{2 t-k} .
$$

This means that for any positive integers $v \geq k+1$ and $u$ satisfying (4) the lemma is admissible with $t=u+v$ and any sufficiently large $c_{5}>0$ (in terms of $k$ and $c_{4}$ ). Choosing $u$ optimally in (4) we conclude that

$$
t=1+v+\left[\frac{\Delta(v)}{2 \varrho}\right]
$$

is admissible; finally, minimizing over $v$ establishes the lemma.

In order to apply Lemma 11 we fix $c_{4}$ such that $c_{3}<c_{4}$, then $c_{5}$ depends only on $k$. In the applications $P$ will be at least $N^{1 / k-o(1)}$, whence $y<R$. As a first corollary, we can state the following mean value estimate for $f$. Note that the same result applies for $h$ in spite of Lemma 8 . 
Lemma 12. Let $t$ be as in Lemma 11. Then we have

$$
\int_{0}^{1}|f(\alpha)|^{2 t} d \alpha \leq N^{2 t / k-1} w^{o(1)}
$$

Proof. Let $\mathcal{B}$ denote the set of vectors $\mathbf{n}=\left(n_{1}, \ldots, n_{2 t}\right)$ with

$$
n_{1}^{k}+\ldots+n_{t}^{k}=n_{t+1}^{k}+\ldots+n_{2 t}^{k} \quad \text { and } \quad 1 \leq n_{1}, \ldots, n_{2 t} \leq N^{1 / k} .
$$

Then

$$
\int_{0}^{1}|f(\alpha)|^{2 t} d \alpha=\sum_{\mathbf{n} \in \mathcal{B}} d_{n_{1}} \ldots d_{n_{2 t}} .
$$

Since $d_{n}>0$ implies that $P(n) \leq y$, we can rewrite this sum as

$$
\int_{0}^{1}|f(\alpha)|^{2 t} d \alpha=\sum_{\mathbf{n} \in \mathcal{C}} d_{n_{1}} \ldots d_{n_{2 t}}
$$

where $\mathcal{C}$ consists of the vectors $\mathbf{n} \in \mathcal{B}$ with

$$
n_{1}, \ldots, n_{2 t} \in \mathcal{A}\left(N^{1 / k}, y\right) \text {. }
$$

Now we can apply Lemma 3 and then Lemma 11 to deduce

$$
\int_{0}^{1}|f(\alpha)|^{2 t} d \alpha \leq w^{o(1)} \sum_{\mathbf{n} \in \mathcal{C}} 1=w^{o(1)} S_{t}\left(N^{1 / k}, y\right) \leq N^{2 t / k-1} w^{o(1)} .
$$

We need an upper estimate for $|f|$ (and $|h|$ ) on the minor arcs $\mathfrak{m}$. Such an estimate is provided by

LEMMA 13. Let $1 / c_{6}=2^{k+1}(F(k)+1)$. Then

$$
\sup _{\mathfrak{m}}|f(\alpha)| \leq \frac{N^{1 / k}}{z^{c_{6}}} w^{o(1)} .
$$

Proof. Let $\alpha \in \mathfrak{m}$. Then Dirichlet's theorem implies the existence of integers $a, q$ with $(a, q)=1$ and $1 \leq q \leq Q$ such that

$$
\left|\alpha-\frac{a}{q}\right| \leq \frac{1}{q Q} \leq \frac{1}{q^{2}}
$$

Since

$$
\mathfrak{m} \subseteq \mathfrak{U} \backslash \mathfrak{M}_{1,1}=[1 / Q, 1-1 / Q),
$$

we have $1 \leq a \leq q$ and then also $q>z$ for otherwise $\alpha$ would be in $\mathfrak{M}$.

Let $t$ be as in Lemma 11 and assume that $t$ is even and minimal. Lemma 11 guarantees the existence of such a $t$ and it also follows that

$$
2 t \leq F(k)+1,
$$

since $F(k)$ is an odd integer. 
We shall apply Hölder's inequality several times without any reference. We have

$$
\begin{aligned}
|f(\alpha)| & =\left|\sum_{\substack{m \leq y \\
l \in \mathcal{L}}} e\left(m^{k} l^{k} \alpha\right)\right| \\
& \leq \sum_{m \leq y}\left|\sum_{l \in \mathcal{L}} e\left(m^{k} l^{k} \alpha\right)\right| \leq y^{1-1 / t}\left(\sum_{m \leq y}\left|\sum_{l \in \mathcal{L}} e\left(m^{k} l^{k} \alpha\right)\right|^{t}\right)^{1 / t} \\
& =y^{1-1 / t}\left(\sum_{\substack{m \leq y \\
l_{1}, \ldots, l_{t} \in \mathcal{L}}} e\left(m^{k}\left(l_{1}^{k}+\ldots+l_{t / 2}^{k}-l_{t / 2+1}^{k}-\ldots-l_{t}^{k}\right) \alpha\right)\right)^{1 / t} .
\end{aligned}
$$

If $N_{0}=\frac{t}{2} \frac{N}{y^{k}}$, and $r(n)$ denotes the number of representations

$$
n=l_{1}^{k}+\ldots+l_{t / 2}^{k}-l_{t / 2+1}^{k}-\ldots-l_{t}^{k}
$$

with

$$
l_{1}, \ldots, l_{t} \in \mathcal{L}
$$

for every integer $n$, then the last inequality implies

$$
\begin{aligned}
|f(\alpha)| & \leq y^{1-1 / t}\left(\sum_{|n| \leq N_{0}} r(n)\left|\sum_{m \leq y} e\left(m^{k} n \alpha\right)\right|\right)^{1 / t} \\
& \leq y^{1-1 / t}\left(\sum_{|n| \leq N_{0}} r(n)^{2} \sum_{|n| \leq N_{0}}\left|\sum_{m \leq y} e\left(m^{k} n \alpha\right)\right|^{2}\right)^{1 /(2 t)} .
\end{aligned}
$$

We shall estimate the inner sums separately. First, $\sum_{|n|<N_{0}} r(n)^{2}$ clearly counts the number of solutions of

$$
\begin{aligned}
l_{1,1}^{k}+\ldots+l_{1, t / 2}^{k}-l_{1, t / 2+1}^{k}- & \ldots-l_{1, t}^{k} \\
& =l_{2,1}^{k}+\ldots+l_{2, t / 2}^{k}-l_{2, t / 2+1}^{k}-\ldots-l_{2, t}^{k}
\end{aligned}
$$

with

$$
l_{1,1}, \ldots, l_{1, t}, l_{2,1}, \ldots, l_{2, t} \in \mathcal{L}
$$

hence, using also Lemma 11,

$$
\sum_{|n| \leq N_{0}} r(n)^{2} \leq S_{t}\left(\frac{N^{1 / k}}{y}, y\right) \leq \frac{N^{2 t / k-1}}{y^{2 t-k}} w^{o(1)} .
$$

Secondly,

(8)

$$
\begin{aligned}
\sum_{|n| \leq N_{0}}\left|\sum_{m \leq y} e\left(m^{k} n \alpha\right)\right|^{2} & \\
& \leq N_{0}^{1-1 / 2^{k-2}}\left(\sum_{|n| \leq N_{0}}\left|\sum_{m \leq y} e\left(m^{k} n \alpha\right)\right|^{2^{k-1}}\right)^{1 / 2^{k-2}} .
\end{aligned}
$$


Estimate the inner sum by applying the Weyl shift $k-1$ times:

$$
\left|\sum_{m \leq y} e\left(m^{k} n \alpha\right)\right|^{2^{k-1}} \ll_{k} y^{2^{k-1}-k} \sum_{\left|h_{1}\right|, \ldots,\left|h_{k-1}\right| \leq y} \min \left(y, \frac{1}{\left\|k ! h_{1} \ldots h_{k-1} n \alpha\right\|}\right),
$$

which yields that

$$
\begin{aligned}
\sum_{|n| \leq N_{0}}\left|\sum_{m \leq y} e\left(m^{k} n \alpha\right)\right|^{2^{k-1}} & \\
& \ll_{k} y^{2^{k-1}-k} \sum_{\substack{|n| \leq N_{0} \\
\left|h_{1}\right|, \ldots,\left|h_{k-1}\right| \leq y}} \min \left(y, \frac{1}{\left\|k ! h_{1} \ldots h_{k-1} n \alpha\right\|}\right) .
\end{aligned}
$$

Let $R(m)$ denote the number of representations of $m$ in the form

$$
m=k ! h_{1} \ldots h_{k-1} n \quad\left(|n| \leq N_{0} ;\left|h_{1}\right|, \ldots,\left|h_{k-1}\right| \leq y\right),
$$

and put $N_{1}=k ! N_{0} y^{k-1}=k ! \frac{t}{2} \frac{N}{y}$. Then the last sum is

$$
\sum_{|m| \leq N_{1}} R(m) \min \left(y, \frac{1}{\|m \alpha\|}\right)
$$

where of course

$$
R(m)=R(|m|) \ll_{k} \begin{cases}N_{1} / y & \text { if } m=0 \\ \tau(m)^{k-1} & \text { if } m \neq 0 .\end{cases}
$$

Hence (9) is, using also Lemma 2,

$$
\begin{aligned}
& \ll_{k} N_{1}+\sum_{1 \leq m \leq N_{1}} \tau(m)^{k-1} \min \left(y, \frac{1}{\|m \alpha\|}\right) \\
& \leq N_{1}+\left(\sum_{1 \leq m \leq N_{1}} \tau(m)^{2 k-2}\right)^{1 / 2}\left(\sum_{1 \leq m \leq N_{1}} \min \left(y, \frac{1}{\|m \alpha\|}\right)^{2}\right)^{1 / 2} \\
& \leq N_{1}+\left(N_{1} \log \left(3 N_{1}\right)^{2^{2 k-2}-1}\right)^{1 / 2}\left(y \sum_{1 \leq m \leq N_{1}} \min \left(y, \frac{1}{\|m \alpha\|}\right)\right)^{1 / 2} .
\end{aligned}
$$

By Lemma 7 we have

$$
\begin{aligned}
\sum_{1 \leq m \leq N_{1}} \min \left(y, \frac{1}{\|m \alpha\|}\right) & \ll\left(\frac{N_{1}}{q}+1\right)(y+q \log q) \\
& \ll k, t \frac{N}{z^{1 / 4}} \log N=\frac{N}{z^{1 / 4}} w^{o(1)}
\end{aligned}
$$


since $N_{1} \ll_{k, t} N / y$ and $z<q \leq N / z^{1 / 4}$. Therefore we see that (9) is

$$
\begin{aligned}
& \ll_{k} N_{1}+\left(N_{1} w^{o(1)}\right)^{1 / 2}\left(y \frac{N}{z^{1 / 4}} w^{o(1)}\right)^{1 / 2} \\
& \ll_{k, t} \frac{N}{y}+\frac{N}{z^{1 / 8}} w^{o(1)}=\frac{N}{z^{1 / 8}} w^{o(1)} .
\end{aligned}
$$

This results in (8) as

$$
\begin{aligned}
\sum_{|n| \leq N_{0}}\left|\sum_{m \leq y} e\left(m^{k} n \alpha\right)\right|^{2} & \leq N_{0}^{1-1 / 2^{k-2}}\left(y^{2^{k-1}-k} \frac{N}{z^{1 / 8}} w^{o(1)}\right)^{1 / 2^{k-2}} \\
& \leq N_{0}^{1-1 / 2^{k-2}}\left(y^{2^{k-1}} \frac{N_{0}}{z^{1 / 8}} w^{o(1)}\right)^{1 / 2^{k-2}} \\
& =\frac{N_{0} y^{2}}{z^{1 / 2^{k+1}}} w^{o(1)}=\frac{N}{y^{k-2} z^{1 / 2^{k+1}}} w^{o(1)}
\end{aligned}
$$

Putting this last inequality and (7) into (6) we get

$$
|f(\alpha)| \leq y^{1-1 / t}\left(\frac{N^{2 t / k-1}}{y^{2 t-k}} w^{o(1)} \frac{N}{y^{k-2} z^{1 / 2^{k+1}}} w^{o(1)}\right)^{1 /(2 t)}=\frac{N^{1 / k}}{z^{1 /\left(2^{k+2} t\right)}} w^{o(1)} .
$$

Now the lemma follows by (5).

5. Proof of the Theorem. At this point we choose $s$, the number of summands in the Theorem. Simply define $s$ as $F(k)$; then we can write it as $2 t+1$ where $t$ has the property formulated in Lemma 11. Consider the function

$$
H(k)= \begin{cases}2^{k}+1 & \text { for } 2 \leq k \leq 4, \\ 4 k & \text { for } k=2^{l}, l \geq 3, \\ \frac{5}{2} k & \text { otherwise. }\end{cases}
$$

We can make the useful observation that

$$
s=F(k) \geq H(k) .
$$

In the only non-trivial case $k \geq 9$ this can be verified by estimating the $\Delta(v)$ 's. For example, very roughly, one can start out from

$$
\Delta(v) \geq(k-2)\left(1-\frac{2}{k}\right)^{v-2},
$$

which follows immediately by induction on $v$. Then, using $1-2 / k>e^{-2.05 / k}$ for $k \geq 43$, one can show that

$$
\min _{k+1 \leq v \leq 2 k+2} \Delta(v)>1
$$


for $k \geq 2+e^{4.1}$. In this case $\varrho<1 /(2 k)$ implies that

$$
\min _{k+1 \leq v \leq 2 k+2}\left[\frac{\Delta(v)}{2 \varrho}\right] \geq k,
$$

and finally that

$$
F(k) \geq 4 k+5 \quad\left(k \geq 2+e^{4.1}\right) .
$$

The remaining cases $(k \leq 62)$ can be checked by hand or by computer.

In order to prove the Theorem, we shall estimate the integrals

$$
J=\int_{\mathfrak{U}} f(\alpha)^{s} e(-N \alpha) d \alpha, \quad I=\int_{\mathfrak{U}} h(\alpha)^{s} e(-N \alpha) d \alpha
$$

simultaneously. First we calculate the contributions of the major arcs $\mathfrak{M}$. Let $\alpha \in \mathfrak{M}(q, a)$. Then $z^{2} \leq y$ together with Lemmata 8 and 10 yields that

$$
\max \left(|f(\alpha)|,\left|q^{-1} S(q, a) f(\beta)\right|\right) \ll L y \quad \text { and } \max \left(|h(\alpha)|,\left|\delta_{q} h(\beta)\right|\right) \ll L y \text {. }
$$

Therefore, using Lemma 1 and the trivial estimate $L y \leq N^{1 / k}$,

$$
\begin{aligned}
& f(\alpha)^{s}=\left(q^{-1} S(q, a)\right)^{s} f(\beta)^{s}+O\left(N^{s / k} y^{-1} z^{2}\right), \\
& h(\alpha)^{s}=\delta_{q} h(\beta)^{s}+O\left(N^{s / k} y^{-1} z^{2}\right) .
\end{aligned}
$$

By the definition of $\mathfrak{M}$,

$$
\begin{aligned}
\int_{\mathfrak{M}} f(\alpha)^{s} e(-N \alpha) d \alpha= & \sum_{\substack{1 \leq a \leq q \leq z \\
(a, q)=1}} \int_{\mathfrak{M}(q, a)} f(\alpha)^{s} e(-N \alpha) d \alpha \\
= & \sum_{\substack{1 \leq a \leq q \leq z \\
(a, q)=1}} \int_{-1 / Q}^{1 / Q}\left\{\left(q^{-1} S(q, a)\right)^{s} e(-N a / q) f(\beta)^{s} e(-N \beta)\right. \\
& \left.+O\left(N^{s / k} y^{-1} z^{2}\right)\right\} d \beta \\
= & \left\{\sum_{\substack{1 \leq a \leq q \leq z \\
(a, q)=1}}\left(q^{-1} S(q, a)\right)^{s} e(-N a / q)\right\} \int_{-1 / Q}^{1 / Q} f(\beta)^{s} e(-N \beta) d \beta \\
& +O\left(Q^{-1} N^{s / k} y^{-1} z^{4}\right) .
\end{aligned}
$$

Now the sum in brackets can be approximated by the singular series

$$
\mathfrak{S}(N)=\sum_{q=1}^{\infty} \sum_{\substack{1 \leq a \leq q \\(a, q)=1}}\left(q^{-1} S(q, a)\right)^{s} e(-N a / q)
$$

which is well known from the theory of the classical Waring problem (cf. $[8])$. We formulate two important properties of this series as 
LEMMA 14. We have

$$
S(q, a) \ll_{k} q^{1-1 / k}
$$

and

$$
1 \ll_{k} \mathfrak{S}(N) \ll_{k} 1 .
$$

Proof. For $k=1$ the statement is obvious. Hence suppose that $k \geq 2$. Then (11) is identical with [8, Theorem 4.2], which also implies the upper bound part of (12). The lower bound in (12) follows by (10) from [8, Theorems 2.5 and 4.6].

The inequality (11) together with (10) implies that

$$
\left(q^{-1} S(q, a)\right)^{s} \ll q^{-5 / 2},
$$

hence

$$
\sum_{\substack{1 \leq a \leq q \\(a, q)=1}}\left(q^{-1} S(q, a)\right)^{s} e(-N a / q) \ll q^{-3 / 2}
$$

always holds, and therefore

$$
\sum_{\substack{1 \leq a \leq q \leq z \\(a, q)=1}}\left(q^{-1} S(q, a)\right)^{s} e(-N a / q)=\mathfrak{S}(N)+O\left(z^{-1 / 2}\right) .
$$

Going back to the integral on $\mathfrak{M}$, we get

$$
\begin{aligned}
\int_{\mathfrak{M}} f(\alpha)^{s} e(-N \alpha) d \alpha= & \mathfrak{S}(N) \int_{-1 / Q}^{1 / Q} f(\beta)^{s} e(-N \beta) d \beta \\
& +O\left(z^{-1 / 2} Q^{-1} N^{s / k}\right)+O\left(Q^{-1} N^{s / k} y^{-1} z^{4}\right),
\end{aligned}
$$

since the integral on the interval $[-1 / Q, 1 / Q]$ is of magnitude $O\left(Q^{-1} N^{s / k}\right)$ by Lemma 8 . By $y^{-1} z^{4}=z^{-1 / 2}$ our formula reads

$$
\int_{\mathfrak{M}} f(\alpha)^{s} e(-N \alpha) d \alpha=\mathfrak{S}(N) \int_{-1 / Q}^{1 / Q} f(\beta)^{s} e(-N \beta) d \beta+O\left(\frac{N^{s / k-1}}{z^{1 / 4}}\right) .
$$

The other part of the integral $J$ can be estimated by Lemmata 12 and 13 as

$$
\left|\int_{\mathfrak{m}} f(\alpha)^{s} e(-N \alpha) d \alpha\right| \leq \sup _{\mathfrak{m}}|f(\alpha)| \int_{\mathfrak{m}}|f(\alpha)|^{2 t} d \alpha \leq \frac{N^{1 / k}}{z^{c_{6}}} w^{o(1)} N^{2 t / k-1} w^{o(1)} .
$$

Summarizing our results concerning $J$ so far we get

$J=\int_{\mathfrak{U}} f(\alpha)^{s} e(-N \alpha) d \alpha=\mathfrak{S}(N) \int_{-1 / Q}^{1 / Q} f(\beta)^{s} e(-N \beta) d \beta+O\left(\frac{N^{s / k-1}}{z^{c_{6}}} w^{o(1)}\right)$,

since $c_{6} \leq 1 / 4$. 
In the same way we get

$$
I=\int_{\mathfrak{U}} h(\alpha)^{s} e(-N \alpha) d \alpha=\int_{-1 / Q}^{1 / Q} h(\beta)^{s} e(-N \beta) d \beta+O\left(\frac{N^{s / k-1}}{z^{c_{6}}} w^{o(1)}\right) .
$$

Now observe that Lemmata 1, 8 and 9 together imply

$$
\begin{aligned}
& \left|\int_{-1 / Q}^{1 / Q} f(\beta)^{s} e(-N \beta) d \beta-\int_{-1 / Q}^{1 / Q} h(\beta)^{s} e(-N \beta) d \beta\right| \\
& =\left|\int_{-1 / Q}^{1 / Q} f(\beta)^{s}\left(1-u(\beta)^{s}\right) e(-N \beta) d \beta\right| \leq s \int_{-1 / Q}^{1 / Q}|f(\beta)|^{s}|1-u(\beta)| d \beta \\
& \quad \leq \pi s \int_{-1 / Q}^{1 / Q}|f(\beta)|^{s}|\beta| U d \beta \leq \pi s \frac{N^{s / k} U}{Q^{2}} \ll_{k} N^{s / k-1} \frac{z^{1 / 2}}{y}=\frac{N^{s / k-1}}{z^{4}} .
\end{aligned}
$$

The expressions obtained for $J$ and $I$ together with this last result and (12) yield that

$$
|J-\mathfrak{S}(N) I| \leq \frac{N^{s / k-1}}{z^{c_{6}}} w^{o(1)} .
$$

Using Lemmata 4,5 and the trivial estimate $h_{n} \geq 0$ for all integers $n$ we are able to estimate the integral $I$. First of all,

$$
\begin{aligned}
& I=\int_{0}^{1} h(\alpha)^{s} e(-N \alpha) d \alpha=\sum_{n_{1}+\ldots+n_{s}=N} h_{n_{1}} \ldots h_{n_{s}} \\
& \geq \sum_{\substack{n_{1}+\ldots+n_{s}=N \\
N /(s+2)<n_{1}, \ldots, n_{s}}} h_{n_{1}} \ldots h_{n_{s}} \\
& \geq\left(\min _{N /(s+2)<n<3 N /(s+2)} h_{n}\right)^{s} \sum_{\begin{array}{c}
n_{1}+\ldots+n_{s}=N \\
N /(s+2)<n_{1}, \ldots, n_{s}
\end{array}} 1 .
\end{aligned}
$$

At this point we choose $0<\lambda<1$ such that $3 /(s+2) \leq \lambda^{k}$, then Lemma 4 implies that

$$
I \geq\left(\frac{L}{U}\right)^{s} \sum_{\substack{n_{1}+\ldots+n_{s}=N \\ N /(s+2)<n_{1}, \ldots, n_{s}}} 1
$$

In order to estimate the last sum, we put

$$
m_{i}=n_{i}-\left[\frac{N}{s+2}\right] \quad \text { for } i=1, \ldots, s .
$$


Then we get

$$
\begin{aligned}
\sum_{\substack{n_{1}+\ldots+n_{s}=N \\
N /(s+2)<n_{1}, \ldots, n_{s}}} 1 & =\sum_{\substack{m_{1}+\ldots+m_{s}=N-s[N /(s+2)] \\
0<m_{1}, \ldots, m_{s}}} 1=\left(\begin{array}{c}
N-s[N /(s+2)] \\
s-1
\end{array}\right) \\
& \geq \frac{1}{(s-1) !}\left(\frac{N}{s+2}\right)^{s-1} \gg_{k} N^{s-1} .
\end{aligned}
$$

Hence, by Lemma 5

$$
I \gg_{k}\left(\frac{L}{U}\right)^{s} N^{s-1} \gg_{k} N^{s / k-1} w^{-s / c_{3}-o(1)},
$$

so that, using (12), we have

$$
|\mathfrak{S}(N) I|>N^{s / k-1} w^{-s / c_{3}-o(1)} .
$$

If $s / c_{3}<(2 / 9) c_{3} c_{6}$, that is, when

$$
c_{3}^{2}>2^{k} 9(F(k)+1) s=2^{k} 9(s+1) s,
$$

then (13) and (14) together yield that

$$
J \geq|\mathfrak{S}(N) I|-|J-\mathfrak{S}(N) I| \geq N^{s / k-1} w^{-s / c_{3}-o(1)} .
$$

Now we recall the definition of $J$ and apply Lemma 3 once more. Since $d_{n}>0$ implies that $P(n) \leq y$, our inequality for $J$ transforms into

$$
\sum_{\substack{n_{1}^{k}+\ldots+n_{s}^{k}=N \\ P\left(n_{1} \ldots n_{s}\right) \leq y}} 1 \geq N^{s / k-1} w^{-s / c_{3}-o(1)} .
$$

This shows that the Theorem holds with any constants

$$
c_{1}>\sqrt{k} s / c_{3}, \quad c_{2} \geq c_{3} / \sqrt{k} .
$$

If we take into account the condition (15) on $c_{3}$, we see that the positive constants $c_{1}, c_{2}$ are admissible whenever

$$
c_{1} c_{2}>s, \quad c_{2}>\sqrt{\frac{2^{k} 9(s+1) s}{k}} .
$$

Note that $s=F(k)$ in these inequalities.

\section{References}

[1] A. Balog and A. Sárközy, On sums of integers having small prime factors $I$, Studia Sci. Math. Hungar. 19 (1984), 35-47.

[2] J. Brüdern, A sieve approach to the Waring-Goldbach problem II. On the seven cubes theorem, Acta Arith. 72 (1995), 211-227.

[3] J. Brüdern and E. Fouvry, Lagrange's four squares theorem with almost prime variables, J. Reine Angew. Math. 454 (1994), 59-96. 
[4] N. G. de Bruijn, On the number of positive integers $\leq x$ and free of prime factors $>y$, Nederl. Akad. Wetensch. Proc. Ser. A 54 (1951), 50-60; II, ibid. 69 (1966), 239-247.

[5] A. Hildebrand, On the number of positive integers $\leq x$ and free of prime factors $>y$, J. Number Theory 22 (1986), 289-307.

[6] A. Hildebrand and G. Tenenbaum, Integers without large prime factors, J. Théor. Nombres Bordeaux 5 (1993), 411-484.

[7] L.-K. Hua, Additive Primzahltheorie, Teubner, Leipzig, 1959; English transl.: Additive Theory of Prime Numbers, Amer. Math. Soc., Providence, 1965.

[8] R. C. Vaughan, The Hardy-Littlewood Method, Cambridge Tracts in Math. 80, Cambridge University Press, 1981.

[9] —, A new iterative method in Waring's problem, Acta Math. 162 (1989), 1-71.

[10] T. D. Wooley, Large improvements in Waring's problem, Ann. of Math. 135 (1992), 131-164.

József u. 9. III. 18.

H-1084 Budapest, Hungary

E-mail: harcos@ludens.elte.hu harcos@math.uiuc.edu 